\title{
PERFIL DEL DOCENTE DE LA CÁTEDRA DE SALUD DEL ADULTO Y ANCIANO SEGÚN LA OPINIÓN DEL ESTUDIANTE DE LA FACULTAD DE ENFERMERÍA DE LA UNIVERSIDAD NACIONAL SAN LUIS GONZAGA DE ICA 2014
}

Profile of the teacher of the adult and elderly health professorship according to the opinion of the student of the faculty of nursing of the National University San Luis Gonzaga from Ica 2014

\author{
Rosa Herlinda Hernández Onofrä, a,c, Amanda María García Aquije ${ }^{1,3, a, c}$, Juana Maribel Arroyo Hernández²,b, \\ Carmen Rosa de la Cruz Injante ${ }^{1, d}$ \\ ${ }^{1}$ Facultad de Enfermería. Universidad Nacional San Luis Gonzaga de Ica, Perú \\ ${ }^{2}$ Facultad de Medicina. Universidad Nacional San Luis Gonzaga de Ica, Perú \\ ${ }^{3}$ Hospital Augusto Hernández Mendoza. Es salud, Ica, Perú \\ a. Licenciada en Enfermería, b. Médico Cirujano, c. Magíster en Salud Pública, d. Estudiante de Enfermería.
}

\section{RESUMEN}

Objetivo: Identificar el perfil del docente de la cátedra de Salud del Adulto y Anciano según la opinión del estudiante de la Facultad de Enfermería de la Universidad Nacional San Luis Gonzaga de Ica. Material y métodos: Estudio descriptivo, de corte transversal. Muestra de 33 alumnos del V y VI Ciclo de la FE-UNICA. Se utilizó la técnica encuesta y como instrumento se utilizó un cuestionario con escala de Valor Likert modificado de la investigadora Peña Guerrero Leslie. Resultados: La situación académica actual de los encuestados fue predominantemente regular (93,9\%). A opinión y expectativa de los alumnos sobre el perfil requerido del docente de la cátedra del Adulto y Anciano fue en el Área Profesional la característica concerniente a demostrar habilidad y destreza en los procedimientos y técnicas de enfermería (32/33) 97,0\% seguido de tener dominio teórico de la disciplina, demostrar técnicas y procedimientos de enfermería a los estudiantes, demostrar dominio en la aplicación práctica del proceso de enfermería (30/33) 90,9\% respectivamente. En el Área Pedagógica fue prevalente el requerimiento para que el docente conozca metodología de la investigación, conocer los objetivos de la asignatura profesional (30/33) 90,9\% respectivamente. En el Área Afectivo Actitudinal, se observó que mayoritariamente se demandan las características: Respetar el horario y la sede asignada por la Institución educativa (30/33) 90,9\%. Conclusiones: El docente del área de Salud del adulto y Anciano debe tener perfil requerido en cuanto a la habilidad de procedimientos y técnicas, y buena metodología de enseñanza.

Palabras claves: Perfil profesional, docencia en enfermería, competencias del docente.

\section{SUMMARY}

Objective: To identify the profile of the teacher of the department of Adult and Elderly Health in the opinion of students of the Faculty of Nursing at the National University of San Luis Gonzaga Ica. Material and Methods: Descriptive, cross-sectional. Sample of 33 students of the V and VI Cycle FE-ONLY. The survey technique was used as an instrument and a questionnaire scale value Likert modified researcher Leslie Peña Guerrero was used. Results: The current academic status of the respondents was predominantly fair (93.9\%). A perception and expectation of students on the required teaching of the Chair of Adult and Elder profile in the Professional Area was the feature concerning demonstrate ability and skill in nursing techniques and procedures (32/33) $97.0 \%$ followed have theoretical mastery of the discipline, demonstrate techniques and procedures of nursing students demonstrated competence in the practical application of the nursing process (30/33) $90.9 \%$ respectively. In the pedagogical area it was prevalent the requirement for teachers to learn research methodology, meet the objectives of the professional course (30/33) 90.9\% respectively. In Attitudinal Affective area, it was observed that mostly demanded characteristics: Respect the schedule and the seat assigned by the educational institution (30/33) 90.9\%. Conclusions: The teaching area and 
Elderly Adult health profile should be required as to the ability of procedures and techniques, and good teaching methodology.

Keywords: Professional profile, teaching nursing, teacher competence.

\section{INTRODUCCIÒN}

Es muy probable que en muchas ocasiones nos hayamos planteado varias preguntas como por ejemplo: ¿Un buen profesor nace o se hace? ¿Será posible aprender a ser un buen profesor? ¿Durante el ejercicio de la docencia solo soy un transmisor de conocimientos? ¿Qué características 0 cualidades deberá tener un buen docente? $Y$ como verán todas estas preguntas han despertado en nuestro grupo un interés, casi intuitivo, que mostramos los docentes sobre la importancia de reflexiones acerca de nuestra función que estamos realizando en las aulas. Para responder estas preguntas partiremos de las siguientes premisas:

- El docente es una persona reflexiva que experimenta en su función situaciones de enseñanza - aprendizaje.

- Un docente informado y capacitado tiene como base conocimientos profundos acerca del proceso enseñanza - aprendizaje.

- Un docente de la cátedras de salud del adulto y anciano deberá responder a un perfil claramente definido, determinado por la meta de alcanzar una acción basada en la calidad profesional y la eficacia pedagógica y la conservación de valores que muestre ante los alumnos un actitud que favorezca el desarrollo humanístico y profesional al alumno.

El proceso de formación de profesional de enfermería, constituye un curriculum de socialización en conocimientos, principios, valores, conciencia sanitaria y habilidades técnicas que se desarrollarán en un campo complejo donde se pondrá de manifiesto los conocimientos científicos, técnicos, éticos y políticos.

Los planes de estudios que se elaboran en la Facultad de Enfermería de la Universidad Nacional San Luis Gonzaga de Ica, tomando en cuenta el remanente teórico, "tienen que ser coherentes con la realidad, con los contenidos y experiencias que se desarrollen en ambientes de aprendizaje que satisfaga las expectativas de los alumnos para que le ayude a formar correctamente en valores, con respeto, autonomía, tolerancia y solidaridad" (1).

El docente de Enfermería en Salud del adulto y Anciano es parte fundamental del equipo que conforma la plana docente de la Facultad de Enfermería de la Universidad Nacional San Luis Gonzaga de Ica. En este contexto como señala el autor "que de su capacidad, experiencia y preparación académica depende en gran parte la efectividad, dinamismo y calidad de la enseñanza, debiendo desarrollar ciertas competencias enfocada en aprender habilidades necesarias para el trabajo, desarrollando modificaciones entre la relación docente - alumno" (2).

Según Demo, las características que debe presentar un docente cuya práctica está centrada en el estudiante es: Investigador, conocimiento propio, habilidades pedagógicas, uso de ambientes virtuales, crítica y enfático (3); por lo que ante las nuevas demandas de los procesos formativos, el nuevo rol docente requiere desempeñar exitosamente varias funciones y tareas no convencionales, orientadas a desarrollar competencias para lograr una docencia semipresencial (4). Al respecto, Waldow señala que el docente debe estar siempre en busca de innovación, proponiendo nuevas formas de actuación que faciliten el 
aprendizaje. Eso significa que los docentes deben reflexionar sobre las cuestiones de enseñanza (5).

El perfil profesional es la descripción de características, potencialidades y competencias, que constituyen los conocimientos, habilidades, destrezas, actitudes y valores que posee un recurso humano determinado para el desempeño de su función dentro de una profesión. La docencia en enfermería desempeña un papel protagónico en la formación de los profesionales de enfermería, pero a la vez se desarrolla en un escenario cargado de inestabilidad ya que pertenece al mundo de la educación y a su vez al mundo de la salud, ambas áreas son socialmente muy compleja y en más de una ocasión no contribuyen al logro de objetivos trazados por las instituciones formadoras (6).

Al respecto, Maitland en su filosofía de la educación en enfermería; hace mención que la meta educacional deberá comprender las necesidades sociales, la adopción individual a los requisitos sociales y al desarrollo de su potencial, Folog concibió un potencial representado por: Conocimientos, habilidades y espíritu, refiriéndose a las características profesionales, psicológicas y actitudes (7).

Las características profesionales están referidas a la capacidad cognoscitiva y desarrollo de actividades intelectuales y al conjunto de funciones y actividades docentes que desarrolla el docente universitario durante la enseñanza y que además refleja una excelente calidad profesional.

En el ejercicio de su profesión las características personales que son conductas referidas a las actividades de actitud $y$ valorización, el hombre, el valor, que suscita el docente (8). La propuesta psicopedagógica de la enseñanza - aprendizaje de enfermería, tiene que proponer modelos psicopedagógicos que orienten el proceso enseñanza aprendizaje a partir de la propia experiencia y el intercambio de opiniones y experiencias de la realidad virtual, nuevas estrategias en función a los campos científicos, tecnológico, acorde con las exigencias sociales (9).

Esta investigación se realizó con el objetivo de identificar el perfil del docente de la cátedra de salud del adulto y anciano según la opinión de los estudiantes de la Facultad de Enfermería de la Universidad Nacional "San Luis Gonzaga" de Ica.

\section{MATERIAL Y MÉTODOS.}

El estudio es de tipo descriptivo, de corte transversal.

Para la recolección de datos se utilizó la técnica encuesta y como instrumento se utilizó un cuestionario con escala de Valor Likert modificado de la investigadora Peña Guerrero Leslie (10).

La recolección de datos se realizó en las aulas de la Facultad de Enfermería de la Universidad Nacional San Luis Gonzaga de Ica, previo permiso de las autoridades correspondientes, se solicitó el consentimiento informado de cada alumno que participo en la investigación.

Con los datos que se obtuvieron se elaboró una base de datos en el software Excel 2010. Para el análisis estadístico se usó el software estadístico SPSS (Statical Package for de Social Sciencias) y el análisis Items del total de encuestas que se aplicó.

Posteriormente se agrupó los ítems que viene a ser características del docente con porcentajes más altos (> 90\%) y se tomó en cuenta para elaborar el perfil del enfermero docente de la cátedra de salud del adulto y anciano según la opinión y expectativa del alumno del V y VI Ciclo de estudios de la carrera de enfermería.

El instrumento se sometió a una prueba piloto utilizando un grupo representativo de 30 alumnos con las mismas características de la población de estudio; donde se validó el coeficiente de correlación ítems - test R (IT- 
TT) y se usó 3 coeficientes de confiablidad Sperman - Brown, Rulon - Gutman, Alfa de Cronbach, para determinar el grado de confiabilidad del instrumento.

\section{RESULTADOS.}

La edad de los estudiantes universitarios de $\mathrm{V}$ y VI ciclo de la Facultad de Enfermería de la UNICA; estuvo comprendido entre los 19 y los 26 años de edad, con una media de 20,4 +/1,5 años; siendo predominante el grupo de los 20 años (12/33) 36,4\%: seguido del grupo de 19 años (10/33) 30,3\%; 21 y 22 años (4/33) $12,1 \%$ respectivamente; 23 años (2/33) $6,1 \%$ y en menor prevalencia el grupo de los 26 años $(1 / 33) 3,0 \%$.

En cuanto al sexo fue predominante el femenino (29/33) $87,9 \%$ y en menor prevalencia el masculino (4/33) $12,1 \%$.

A la pregunta ¿Ha repetido alguna asignatura? Respondió anatomía (3/33) 9,1\%; fisiología y biología, microbiología y parasitología, farmacología (1/33) 3,0\% respectivamente; en los demás casos negaron esta posibilidad (27/33) $81,8 \%$

Se encuestó predominante a estudiantes de enfermería del quinto ciclo (22/33) $66,7 \%$ y en menor prevalencia sexto ciclo (11/33) $33,3 \%$.

La situación académica actual de los encuestados fue regular (31/33) $93,9 \%$ y en menor prevalencia $(2 / 33) 6,1 \%$ (tabla $N^{\circ} 1$ )

Tabla $\mathbf{N}^{\circ}$ 01: Datos generales de 33 estudiantes universitarios de la Facultad de Enfermería encuestados para pedir su opinión acerca de las características que debe poseer el Docente de la cátedra de Salud del Adulto y Anciano

\begin{tabular}{|c|c|c|}
\hline \multirow{2}{*}{\multicolumn{3}{|c|}{$\begin{array}{l}\text { (Media 20,4 } \pm \\
1,5 \text { años) }\end{array}$}} \\
\hline & & 303 \\
\hline 20 & 12 & 36.4 \\
\hline 21 & 4 & 12,1 \\
\hline 22 & 4 & 12,1 \\
\hline 23 & 2 & 6,1 \\
\hline 26 & 1 & 3,0 \\
\hline \multicolumn{3}{|l|}{ Sexo } \\
\hline Femenino & 29 & 87,9 \\
\hline Masculino & 4 & 12,1 \\
\hline \multicolumn{3}{|c|}{ ¿Ha repetido alguna asignatura profesional? } \\
\hline Anatomía & 3 & 9,1 \\
\hline $\begin{array}{l}\text { Fisiología y } \\
\text { biología }\end{array}$ & 1 & 3,0 \\
\hline Microbiología & 1 & 3,0 \\
\hline & & \\
\hline Farmacología & 1 & 3,0 \\
\hline No & 27 & 81,8 \\
\hline \multicolumn{3}{|c|}{ ¿Qué ciclo cursa actualmente? } \\
\hline$\checkmark$ ciclo & 22 & 66,7 \\
\hline VI ciclo & 11 & 33,3 \\
\hline \multicolumn{3}{|c|}{ Situación académica actual } \\
\hline Regular & 31 & 93,9 \\
\hline Reingresante & 2 & 6,1 \\
\hline
\end{tabular}

A opinión y expectativa de los alumnos de $\mathrm{V}$ y VI ciclo de la Facultad de Enfermería el perfil requerido prevalente del docente de la cátedra del Adulto y Anciano fue en el AREA PROFESIONAL para demostrar habilidad $y$ destreza en los procedimientos y técnicas de enfermería de la disciplina que enseña (32/33) $97,0 \%$ seguido de tener dominio teórico de la disciplina que enseña, demostrar las técnicas y procedimientos de enfermería a los estudiantes de enfermería, demostrar dominio en la aplicación práctica del proceso de enfermería (30/33) 90,9\% respectivamente; en el AREA PEDAGOGICA fue prevalente el requerimiento para que el docente conozca metodología de la investigación, conocer los objetivos de la asignatura profesional en la que participa (30/33) 90,9\% respectivamente y en el AREA AFECTIVO ACTITUDINAL para respetar el horario y la sede asignada por la Institución educativa

Tabla N 02: Características del docente con porcentajes altos $(>90,0 \%)$ según la opinión y expectativa del alumno del $\mathrm{V}$ y VI ciclo de estudios de la Facultad de Enfermería 


\begin{tabular}{|c|c|c|c|c|c|c|c|}
\hline \multirow{2}{*}{$\begin{array}{l}\text { Dimensi } \\
\text { ones }\end{array}$} & \multirow[t]{2}{*}{ Reactivos } & \multicolumn{2}{|c|}{ Poco } & \multicolumn{2}{|c|}{ Medianam } & \multicolumn{2}{|c|}{ Totalmente } \\
\hline & & $\mathbf{N}$ & $\%$ & $\mathbf{N}$ & $\%$ & $\mathbf{N}$ & $\%$ \\
\hline \multirow{7}{*}{$\begin{array}{l}\text { Área } \\
\text { profesio } \\
\text { nal }\end{array}$} & $\begin{array}{l}\text { Tener dominio } \\
\text { teórico }\end{array}$ & 1 & 3,0 & 2 & 6,1 & 30 & 90,9 \\
\hline & $\begin{array}{l}\text { Demostrar } \\
\text { habilidad }\end{array}$ & - & - & & & & \\
\hline & & & & 1 & 3,0 & 32 & 97,0 \\
\hline & $\begin{array}{l}\text { técnicas de } \\
\text { enfermería }\end{array}$ & & & & & & \\
\hline & Demostrar las & - & - & 3 & 9,1 & 30 & 90,9 \\
\hline & $\begin{array}{l}\text { técnicas y } \\
\text { procedimientos } \\
\text { de enfermería a } \\
\text { los estudiantes }\end{array}$ & & & & & & \\
\hline & $\begin{array}{l}\text { Demostrar } \\
\text { dominio en la } \\
\text { aplicación } \\
\text { práctica }\end{array}$ & - & - & 3 & 9,1 & 30 & 90,9 \\
\hline \multirow{3}{*}{$\begin{array}{l}\text { Área } \\
\text { pedagóg } \\
\text { ica }\end{array}$} & $\begin{array}{l}\text { Conocer } \\
\text { metodología }\end{array}$ & - & - & 3 & 9,1 & 30 & 90,9 \\
\hline & Conocer los & & & & & & \\
\hline & $\begin{array}{l}\text { objetivos de la } \\
\text { asignatura }\end{array}$ & 1 & 3,0 & 2 & 6,1 & 30 & 90,9 \\
\hline $\begin{array}{c}\text { Area } \\
\text { afectivo } \\
\text { actitudin } \\
\text { al }\end{array}$ & $\begin{array}{l}\text { Respetar el } \\
\text { horario y la sede } \\
\text { asignada }\end{array}$ & - & - & 3 & 9,1 & 30 & 90,9 \\
\hline
\end{tabular}

A los reactivos planteados en la dimensión AREA PROFESIONAL los encuestados respondieron como totalmente requerida para el perfil del docente universitario de Enfermería en la cátedra de Salud del Adulto y Anciano con el (27/33) $81,8 \%$ y medianamente requerida $(3 / 33) 18,2 \%$ (tabla $\mathrm{N}^{\circ} 3$ )

Tabla $N^{\circ}$ 03: Perfil del docente en la cátedra de Salud del Adulto y Anciano en la dimensión área profesional a opinión de estudiantes de Enfermería

\begin{tabular}{lcc}
\hline \multicolumn{1}{c}{ Área profesional } & Frecuencia & Porcentaje \\
\hline $\begin{array}{l}\text { Medianamente } \\
\text { requerida (19-37) }\end{array}$ & 6 & 18,2 \\
$\begin{array}{l}\text { Totalmente } \\
\text { requerida (38-56) }\end{array}$ & 27 & 81,8 \\
\hline Total & 33 & 100,0 \\
\hline
\end{tabular}

A los reactivos planteados en la dimensión AREA PEDAGÓGICA los encuestados respondieron como totalmente requerida para el perfil del docente universitario de Enfermería en la cátedra de Salud del Adulto y Anciano con el (27/33) $81,8 \%$ y medianamente requerida (6/33) $18,2 \%$ (tabla $N^{\circ} 04$ )

Tabla $\mathbf{N}^{\circ}$ 04: Perfil del docente en la cátedra de Salud del Adulto y Anciano en la dimensión área pedagógica a opinión de estudiantes de Enfermería

\begin{tabular}{lcc}
\hline \multicolumn{1}{c}{ Área pedagógica } & Frecuencia & Porcentaje \\
\hline $\begin{array}{l}\text { Medianamente } \\
\text { requerida (23-46) }\end{array}$ & 6 & 18,2 \\
$\begin{array}{l}\text { Totalmente } \\
\text { requerida (47-68)* }\end{array}$ & 27 & 81,8 \\
\hline
\end{tabular}

A los reactivos planteados en la dimensión AREA AFECTIVO ACTITUDINAL IOS encuestados respondieron como totalmente requerida para el perfil del docente universitario de Enfermería en la cátedra de Salud del Adulto y Anciano con el (28/33) $84,8 \%$ y medianamente requerida (5/33) $15,2 \%$ (tabla $N^{\circ} 05$ )

Tabla N 05: Perfil del docente en la cátedra de Salud del Adulto y Anciano en la dimensión características afectivo actitudinales del docente a opinión de estudiantes de Enfermería

\begin{tabular}{lcc}
\hline \multicolumn{1}{c}{$\begin{array}{c}\text { Área afectivo } \\
\text { actitudinal }\end{array}$} & Frecuencia & Porcentaje \\
\hline $\begin{array}{l}\text { Medianamente } \\
\text { requerida (19-37) }\end{array}$ & 5 & 15,2 \\
$\begin{array}{l}\text { Totalmente } \\
\text { requerida (38-56) }\end{array}$ & 28 & 84,8 \\
\hline Total & 33 & 100,0 \\
\hline
\end{tabular}

\section{DISCUSIÓN}

Determinar el Perfil del Docente Universitario es un proceso técnico multidisciplinario en el que participan sociólogos, psicólogos, pedagogos, y diversos especialistas en Educación Superior. Participan además profesionales capaces de realizar un diagnóstico situacional del medio en el que se va desempeñar el docente, la malla curricular de la cual el determinado perfil será un componente más, y deberá actuar como parte de un todo que responde a objetivos mayores que se concatenan con los que a nivel de una determinada asignatura se cumplan a cabalidad.

En el presente trabajo, no obstante hemos deseado conocer el punto de vista del alumnado, quien consideramos no puede estar exento de este debate, y no nos cabe duda, puede aportar información muy valiosa respecto de una labor que no podemos describir los colegas al no estar in situ en el día a día del desenvolvimiento docente en el continuo enseñanza - aprendizaje. En ese sentido, nos propusimos Determinar cuál sería 
el Perfil del Docente de la Cátedra de Salud del Adulto y Anciano de la Facultad de Enfermería de la Universidad Nacional San Luis Gonzaga de Ica

La edad de los estudiantes universitarios que participaron en la investigación estuvo comprendida entre los 19 y los 26 años de edad, con una media de 20,4. En cuanto al sexo fue ampliamente predominante el femenino (87,9\%). Para Manuela A. Fernández, et al. (2012) (11) el género de los alumnos en estudios similares no varía significativamente respecto a las preferencias $u$ opiniones del perfil del docente universitario. $\mathrm{La}$ interrogante $¿ \mathrm{Ha}$ repetido alguna asignatura? mayoritariamente negaron esta posibilidad $(81,8 \%)$. Se encuestó de manera predominante a estudiantes de enfermería del quinto ciclo $(66,7 \%)$ y en menor prevalencia del sexto ciclo. La situación académica actual de los encuestados fue predominantemente regular $(93,9 \%)$.

A opinión y expectativa de los alumnos sobre el perfil requerido prevalente del docente de la cátedra del Adulto y Anciano fue en el Área Profesional la característica concerniente a demostrar habilidad y destreza en los procedimientos y técnicas de enfermería de la disciplina que enseña (32/33) 97,0\% seguido de tener dominio teórico de la disciplina que enseña, demostrar las técnicas y procedimientos de enfermería a los estudiantes de enfermería, demostrar dominio en la aplicación práctica del proceso de enfermería (30/33) 90,9\% respectivamente; al respecto coinciden estos resultados parcialmente con los obtenidos por Asmat et al.,(Lima, 1988) (12) quienes en un estudio similar reportaron los alumnos intervenidos la necesidad de contar con docentes que tengan un amplio dominio de su curso. En el Área Pedagógica fue prevalente el requerimiento para que el docente conozca metodología de la investigación, conocer los objetivos de la asignatura profesional en la que participa (30/33) 90,9\% respectivamente. Al respecto
Asmat et al.,(Lima, 1988) (12) reportan características muy similares a nuestro estudio tales como: necesidad de prepararse en pedagogía universitaria, no debe improvisar su clase, conocer objetivos, ser especialista en la asignatura que enseña, conocer la metodología de investigación, aplicar el proceso de enfermería, definir el objetivo de las acciones que espera que sus alumnos realicen. Por su parte también la investigación de Gloria Vásquez (Lima 1987) (13) reporta necesidades de características afines a las determinadas en nuestro estudio tales como dominar metodología de investigación para la asesoría de tesis, aplicar el proceso de enfermería con metodología unificada de enseñanza a los estudiantes.

En el Área Afectivo Actitudinal comprendida en nuestro estudio, se observó que mayoritariamente se demandan las características: Respetar el horario y la sede asignada por la Institución educativa (30/33) 90,9\%. Al respecto Asmat et al., (Lima, 1988) (12) reportan la característica: llevarse bien con los alumnos. Mientras que Gloria Vásquez (Lima 1987) (13) reporto en esta área las características: mantener permanente el diálogo, ser el transmisor del modelo profesional, contribuir a fortalecer la imagen del profesional, conservar la comunicación con los miembros del equipo de salud donde acuden los estudiantes, promover el intercambio institucional.

\section{CONCLUSIONES}

1. Los Estudiantes entre el quinto y sexto ciclo de la Facultad de Enfermería de la UNICA consideran que en el área técnico profesional, el docente de enfermería de la cátedra de Salud del Adulto y Anciano requiere totalmente las siguientes características: demostrar 
habilidad y destreza en los procedimientos y técnicas de enfermería de la disciplina que enseña, tener dominio teórico de la disciplina que enseña, demostrar las técnicas y procedimientos de enfermería a los estudiantes de enfermería, y demostrar dominio en la aplicación práctica del proceso de enfermería.

2. Los Estudiantes entre el quinto y sexto ciclo de la Facultad de Enfermería de la UNICA consideran que en el área técnica pedagógica, el docente de enfermería de la cátedra de Salud del Adulto y Anciano, requiere totalmente las siguientes características: conocer metodología de la investigación y conocer los objetivos de la asignatura profesional en la que participa.

3. Los Estudiantes entre el quinto y sexto ciclo de la Facultad de Enfermería de la UNICA consideran que en el área afectivo actitudinal, el docente de enfermería de la cátedra de Salud del Adulto y Anciano requiere totalmente las siguientes características: Respetar el horario y la sede asignada por la Institución educativa.

4. Según la opinión de los encuestados no se presentaron diferencias significativas entre las áreas contempladas y el requerimiento en mayor o menor grado de alguna en particular.

\section{RECOMENDACIONES}

Podemos proponer respaldados en la evidencia generada que se realice estudios de investigación en busca de las características que posee en la actualidad en general la plana completa de docentes de la Facultad de Enfermería de la UNICA a fin de obtener una situación diagnóstica que permita a las autoridades identificar los componentes que pueden reforzarse en el corto, mediano y largo plazo; ya sean estos comprendidos en el Área Profesional, Pedagógica o Afectivo actitudinal.

\section{Correspondencia:}

Mg. Rosa Hernández Onofra

Correo electrónico: rosaherono@hotmail.com

Celular:

\section{REFERENCIAS BIBLIOGRÁFICAS}

1. Castrillon M. Pensando en la formación de futuros profesionales de enfermería en América Latina. Rev Invest educ enferm. 2008 marzo-agosto, Antioquía Colombia; 26 (2 supl): 114-121.

2. Inahiá P. Reflexiones sobre la competencia docente e la enseñanza de enfermería. Brasil Rev. Esc. Enferm USP .2007; 41(4) (Internet) (citado 24 Ene 2014). Disponible en: www.ee.usp.br/reeusp/

3. Demo P. Educación y conocimiento: relación necesaria, insuficiente y controversia. 3a ed. Brasil: Vozces; 2000. p. 40

4. Montero P. Desafíos para la profesionalización del nuevo rol docente universitario. Rev Aval pol públ Educ. Brasil. 2007; 15 (56) (Interne) (citado 30 Nov de 2013) Disponible en: http://www.scielo.br/pdf/ensaio/v15n56 la03v1556.pdf.

5. Waldow R. Estrategia de enseñanza en enfermería: enfoque en el cuidado y el pensamiento. $2^{\mathrm{a}}$ ed. Lima, Perú: Vozces; 2004. p.9-10

6. Cuarto Congreso Internacional de Pensamiento Latino americano. La formación de docentes en América Latina y en Colombia. San Juan de Pasto; 2004 Octubre 10; Nariño: Universidad de Nariño; Colombia; 2004.

7. Kérouac S, Pepin J, Ducharne F, Mayor F. El pensamiento enfermero. Madrid, España: Mason; 2000.

8. Watson J. Enfermería: ciencia humana y cuidada humano. En: Waldow R. Cuidar: expresiónhumanizadora de la enfermera. 1a ed. Lima, Perú: Vozces; 2008.p. 27-36

9. Marsh. H. y Bailey, M. Multidimensional students' evaluations of teaching effectiveness. Journal of Higher Education, Sidney.; 1993.

10. Peña L. Características requeridas en el docente de enfermería según opinión de la 
comunidad educativa de la UNMSM 20012003 (tesis). Lima, Perú: Facultad de Medicina, EAP DE Enfermería, Universidad Nacional Mayor de San Marcos; 2003.

11. Fernández $\mathbf{M}$; Gonzáles $\mathbf{S}$. El perfil del buen docente universitario, una aproximación en función del sexo del alumnado. España, .Rev. Docencia Universitaria.2012; 10(2):. 237-249.

12. Asmat E. Perfil professional de la docente de enfermería según opinión de estudiantes y docentes de la Escuela Nacional de Enfermería Arzobispo Loayza. UPCH. Perú; 1988

13. Vásquez G. Características que debe reunir el docente de Enfermería según opinión de docentes y estudiantes de Enfermería de la UNMSM. Universidad Nacional Mayor de San Marcos. Lima, Perú; 1987.

Recibido: 20/08/2016

Aprobado para Publicación: 23/11/2016 